\title{
Reforma agraria, conflictos por la tierra y cambios en el poblamiento: caso de estudio de la mesorregión de Presidente Prudente en el Estado de São Paulo (Brasil)
}

\author{
Agrarian reform, land conflicts, and population changes: a case study \\ of the Presidente Prudente mesoregion in the State of São Paulo (Brazil)
}

\author{
Maryna Vieira Martins Antunes ${ }^{1}$ (D) \\ Samuel Esteban Rodríguez $2^{2 *}$ (iD
}

\section{Resumen}

En la actualidad, existen en Brasil procesos de creación de asentamientos rurales llevados a cabo por políticas gubernamentales. En este trabajo se analiza el impacto de estas políticas sobre el hábitat rural, tomando como caso de estudio la mesoregión de Presidente Prudente en São Paulo (Brasil). El trabajo realizado consiste en un análisis del poblamiento a escala municipal basado en el índice de Demangeon, un índice clásico para estudiar el tipo de hábitat. Las fuentes de datos son la Fundación del Instituto de Tierras de São Paulo y el Instituto Brasileño de Geografía y Estadística. En el trabajo se comparan dos escenarios: el primero analiza el poblamiento tomando como base las entidades preexistentes a la intervención del gobierno; el segundo analiza el poblamiento actual considerando los nuevos núcleos de población. Este análisis permite concluir que la intervención del gobierno ha aumentado la dispersión del poblamiento, especialmente en las áreas próximas a la frontera con los estados de Mato Grosso del Sur y Paraná; zonas de reforma agraria y dónde se mantienen conflictos por la titularidad de la tierra entre los latifundistas y el Movimiento de los Trabajadores Rurales Sin Tierra. hábitat.

Palabras clave: reforma agraria; Presidente Prudente, São Paulo (Brasil); poblamiento; Demangeon;

\begin{abstract}
Brazilian government policies are leading to processes for the creation rural settlements. This paper analyses the impact of these policies on the rural habitat; for this, the administrative mesoregion of Presidente Prudente in the State of São Paulo (Brazil) has been taken as a case study. The work carried out consists of an analysis of the system of settlements in the municipalities of the region - and using as data sources the São Paulo State Land Institute Foundation and the Brazilian Institute of Geography and Statistics. The Demangeon index, a classic index to study settlement distribution, is used to compare two scenarios: the system of settlements before government intervention; and the current system of settlements considering both old and new population centres. The analysis carried out enables us to conclude that government intervention has increased the settlement dispersion. This process has been especially acute in the areas near the border with Mato Grosso do Sul and the state of Paraná. These agrarian reform areas have seen land conflicts between landowners and the Landless Workers' Movement.

Keywords: Agrarian reform; Presidente Prudente, São Paulo (Brazil); Brazil; system of settlements; Demangeon; habitat.

1 Universidade Estadual Paulista, Faculdade de Ciências e Tecnologia, Câmpus de Presidente Prudente, Coordenação de Aperfeiçoamento de Pessoal de Nível Superior, Brasil. antunes.maryna@gmail.com

2 Grupo de Estudios de Ordenación del Territorio (GEOT) - Universidad de Zaragoza, España. sestebanr@unizar.es. *Autor para correspondencia.




\section{Introducción}

\subsection{El problema del reparto de la tierra}

La concentración de tierras en Brasil es un problema estructural que tiene sus orígenes en el propio proceso de formación del país. En el siglo XVI se constituyen, como divisiones de la colonia, las capitanías hereditarias. Las capitanías fueron donaciones de grandes porciones de tierra por parte de la corona portuguesa a los que serían nombrados gobernadores de las mismas. A su vez, ante la necesidad de poblar el territorio, se donó parte de las tierras de la capitanía para su explotación agraria (Ebling, 2009). Estas propiedades se llamaron sesmarias y con ellas se instauró la agricultura de plantación en Brasil. Este sistema agrícola se caracterizaba por grandes propiedades, monocultivo y uso de mano de obra esclava (George, 1974).

Las plantaciones requerían importantes contingentes de trabajadores; sin embargo, solían localizarse en zonas poco pobladas y se recurrió al tráfico de esclavos (Molinero, 1990). La presencia de poblaciones de diferentes orígenes convirtió a las plantaciones en "una amalgama racial y cultural" (Molinero, 1990, p. 151) lo que ha pasado a ser un elemento característico del país; a la par, la gran cantidad de personas ocupadas en ellas derivaría en uno de los rasgos más llamativos de la estructura de la tierra brasileña: mucha tierra con pocos dueños y mucha gente sin tierra. En Brasil, el resultado del periodo colonial fue la formación de grandes latifundios (Osorio, 2006).

El año de la independencia de Brasil, 1822, se deroga la ley de sesmarias y la ocupación se convierte en la única forma de adquirir la propiedad de las tierras vacantes (Ebling, 2009). En este contexto, la ocupación de tierras devolutas, es decir, públicas, fue utilizada tanto en el contexto de una economía de subsistencia por pequeños productores a los que no se había concedido una sesmaria (Germani, 1997) como por grandes propietarios de plantaciones (Ebling, 2009).

Frente a las donaciones y la ocupación de tierras de los periodos previos, en 1850 se aprueba una Ley de Tierras (Ley $\mathrm{n}^{\circ} 601$, de 18 de septiembre de 1850) que establece que el acceso a las tierras devolutas fuera a través de compra (Osorio, 2006). Esta norma, puesta en marcha pocos años antes de la abolición de la esclavitud en 1888, impidió el acceso a la tierra a millones de personas; destacando los antiguos esclavos que, al carecer de posesiones y de medios para comprarlas, permanecieron en el cautiverio de la tierra (Martins, 1996). Es decir, personas que, nominalmente, dejaron de ser esclavas pero que tuvieron que continuar trabajando como asalariados de la misma plantación. A este problema, se sumó el hecho de que hubo ocupantes de tierras que no pudieron regularizar sus títulos (Osorio, 2006).

El reglamento que desarrollaba la ley de tierras instituyó la elaboración de un catastro que no funcionó, pues fue una importante vía de entrada de fraudes en la demarcación de las tierras (Osorio, 2006). Adicionalmente, la necesidad de contar con un documento acreditativo de la compra-venta de la tierra para ser propietario dio origen a una práctica denominada grilagem ${ }^{3}$ que consistía en falsificar los documentos de propiedad. Con estos documentos falsificados, en ocasiones, se vendía a los pequeños campesinos terrenos que poseían de hecho (Oliveira, 2015) En otros casos, el grilagem ha sido utilizado para el acaparamiento de tierras; algo común hasta en el día de hoy y que, a menudo, se asocia con prácticas violentas contra pequeños propietarios, trabajadores rurales y pueblos indígenas (Fernandes, 1994). Estas prácticas frecuentemente se vinculan con la expansión de la frontera agrícola y el agro-negocio: casos como la invasión de la Amazonia no pueden disociarse de este interés por la tierra que subyace tras ellas (Oliveira, 2015; Bernardes y Maldonado, 2017).

El sistema de plantación, que todavía está vigente, puede considerarse, dentro de la agricultura de especulación, como "la forma superlativa de la agricultura de mercado" (George, 1974, p. 257). Sin embargo, ha pasado de sus primeras fases, centradas en la caña de azúcar y el algodón a otros cultivos como el café, con gran importancia en el Estado de São Paulo (Márquez, 1992) o, en las últimas décadas, la soja y los agrocombustibles (Oliveira, 2009).

Es frecuente que estas grandes explotaciones hayan contado con la complicidad del Estado a la hora de tomar la posesión del suelo; "con mucha frecuencia se utilizan tierras en las que la renta territorial es nula: tierras desocupadas, tierras confiscadas o adquiridas a precios extremadamente bajos" (George, 1974, p. 263). Ejemplo de ello fueron las numerosas compañías cafeteras de São Paulo que se extendieron sobre tierras devolutas desplazando a los pequeños productores que las ocupaban (Osorio, 2006).

3 La palabra grilagem proviene del método de falsificación utilizado; frecuentemente se envejecían los documentos en cajones junto a grillos para darles una apariencia confiable. 


\subsection{Demandas sociales y políticas de reforma agraria}

El proceso histórico de atribución de la propiedad descrito ha provocado la existencia de un gran número de títulos de propiedad controvertidos y, sobre todo, ha generado grandes contingentes de trabajadores pobres en el campo (Wanderley, 2014). Esto se ha visto reforzado por el modelo de la agricultura de plantación, que ha dado lugar a "la paradoja de coexistir una agricultura especulativa y altamente especializada con espacios de agricultura de subsistencia" (Márquez, 1992, p. 121). Esta situación ha favorecido la aparición de entidades como el Movimiento de los Trabajadores sin Tierra (MST) que, desde su fundación en la década de 1980, reclama una reforma agraria con reparto de la tierra entre la población (Fernandes, 1994).

Sin embargo, en Brasil, la lucha por la tierra no es algo nuevo. Históricamente han sido frecuentes los conflictos relacionados con esta cuestión desde la lucha por la libertad de los esclavos. Pese a esto, a la par que se produjo una intensificación de los monocultivos y una expansión de la agroindustria, en la década de 1970, la dictadura militar reprimió los movimientos campesinos, casi llevándolos a su extinción. Posteriormente, en la década de 1980 se consolida un modelo agroindustrial de exportación, a la par que se produce la redemocratización del país. En estas circunstancias, se dio un contexto propicio para el crecimiento de los movimientos sociales, de manera organizada y con un objetivo claro: la reforma agraria (Fernandes, 2008).

La acepción más extendida de reforma agraria implica una redistribución de la propiedad, especialmente la de los latifundios, entre los campesinos sin tierra (Ilbery, 1985); esto supone una modificación de las relaciones económicas y sociales (George, 1974). El instrumento de la reforma agraria es la indemnización al gran propietario, cuando éstos no son indemnizados se habla de revoluciones agrarias (Márquez, 1992). La reforma agraria puede incluir mejoras técnicas de los sistemas agrarios y puesta en explotación de nuevas tierras, mediante procesos de roturación y colonización (Márquez, 1992). Sin embargo, en un país caracterizado por la presencia de grandes latifundios, la reforma agraria, tal como afirma Mattei (2012), implica una democratización del acceso a la tierra.

En respuesta a las demandas sociales, desde mediados de la década de 1980 y, sobre todo, de los años 1990, el gobierno federal pone en marcha políticas de reforma agraria y creación de asentamientos rurales. Desde los años 1960 se preveía la expropiación de tierras que no cumplían un uso social, es decir, aquellas donde no se adoptara un uso racional de acuerdo con el Estatuto de la Tierra, Ley n ${ }^{\circ} 4.504$, de 30 de noviembre de 1964. Esta norma fue actualizada por la ley 8.629, de 25 de febrero de 1993; pues poco o casi nada se había hecho de reforma agraria en este tiempo (Peixoto, 2017). A lo largo de los años 1990 y 2000 se han adoptado medidas que, aunque no han sido capaces de corregir el problema de los trabajadores sin tierra de forma satisfactoria para la totalidad de las partes, han incrementado el número de asentamientos creados en relación con procesos de reforma (Cosme, 2016). Sin embargo, Fernandes y Girardi (2008) ponen de relieve que lo que ocurre en Brasil puede entenderse más como una política de creación de asentamientos que como una reforma agraria en sentido amplio: junto a la puesta en marcha de nuevos asentamientos rurales, hay una expansión de la frontera agrícola hacia zonas como la Amazonia y el Cerrado, lo que está generando nuevos latifundios, de modo que la tierra permanece concentrada.

\subsection{El hábitat rural}

El hábitat, ..., ha sido estudiado tradicionalmente contraponiendo las dos formas extremas: hábitat disperso y hábitat concentrado. El disperso está formado por casas de campo o labranza que se distribuyen de forma aislada por el espacio agrario y sirven para dar cobijo a los trabajadores agrarios y sus familias, sus animales y sus aperos. Cuando varias de estas casas se unen en agrupamientos más o menos importantes, en medio de los campos de cultivo, hablamos de hábitat concentrado. Entre estos dos casos extremos tan claros existe gran variedad y a veces resulta difícil establecer el límite entre ambos. (Aguilera, Borderías, Gonzáles y Santos, 1991, p. 179)

Entre los factores clásicos que se han utilizado para explicar el agrupamiento y la dispersión del hábitat rural destacan los planteados por George (1974): factores naturales, tales como la disposición de los puntos de agua y manantiales. Factores históricos y defensivos, como puede ser el efecto del grupo a la hora de articular la defensa de un enclave. Además de estos añade, los factores sociales y económicos, entre los que destaca la forma de propiedad y la organización del trabajo. Es importante en este sentido el papel de la dispersión de las propiedades de cada familia o pequeño propietario a la hora de convertir el núcleo rural en un lugar central en el que convergen los caminos entre estas parcelas, lo que favorecería 
un hábitat concentrado, frente a esto, en los casos donde la explotación familiar tiene las dimensiones suficientes se tiende a la dispersión (George, 1974).

En el caso de la agricultura de plantación cobran gran importancia los factores económicos, pues, tradicionalmente ha requerido de la agrupación de mano de obra permanente. Esto la ha convertido en una economía caracterizada por un hábitat eminentemente concentrado, con pueblos grandes y depauperados (George, 1974). Por otro lado, en caso de requerir temporeros para determinadas actividades de carácter estacional, se adopta el campamento temporal como solución habitacional para alojarlos (George, 1974). Sin embargo, hoy crece la mecanización del trabajo en el campo, lo que está contribuyendo a un progresivo vaciado demográfico del medio rural (Navarro, 2017). En la actualidad, cabe destacar dentro de la agricultura de plantación el cultivo de la caña de azúcar en São Paulo (Barreto, 2012) y la soja o el café en la región del Cerrado (Lima, Locatel y da Silva, 2012; Ortega y Jesus, 2011).

Por otro lado, es necesario tener en cuenta que, todavía, en el interior de Brasil existen grandes áreas con densidades de población muy bajas, pese a contar con alta riqueza natural. La acción planificada del Estado podría atraer población a estas regiones, como sucedió con la creación de Brasilia (D'Entremont, 1997). En ámbitos rurales, los procesos de colonización o conquista de nuevos espacios agrícolas también pueden suponer una expansión del poblamiento. Tanto la colonización de nuevas tierras como las reformas pueden acarrear profundas modificaciones en los componentes de los sistemas agrarios (Márquez, 1992) y, a su vez, esta reorganización territorial de la actividad económica puede tener un efecto en el sistema de asentamientos (D'Entremont, 1997).

Teniendo en cuenta los aspectos que se han comentado, las políticas de reforma agraria y de creación de asentamientos rurales que se están llevando a cabo en Brasil desde los años 90 han podido tener un impacto importante sobre el territorio. La creación de nuevas entidades de población es fundamental para la ocupación de espacios y la expansión de la ecúmene. En este sentido, el impacto iría más allá de una mera modificación de los títulos de propiedad, pues se trata de cambios en el poblamiento de las zonas afectadas.

\subsection{Objetivos de trabajo}

Para valorar si los procesos de colonización y reforma agraria que se están llevando a cabo en Brasil tienen implicaciones sobre el poblamiento, en este trabajo se plantea un análisis comparativo del tipo de hábitat antes y después de las intervenciones llevadas a cabo desde 1990; para ello se ha tomado como caso de estudio la mesoregión de Presidente Prudente, en el Estado de São Paulo. Se trata de una zona de ocupación reciente con problemas de acaparamiento y titularidad de las tierras, presencia de una gran cantidad de trabajadores sin tierra, actividad del MST, presencia de procesos de expropiación en la actualidad y, también, de creación de nuevos asentamientos rurales.

El artículo se organiza de la siguiente manera: en el apartado de metodología se caracteriza el área de estudio y se detalla el procedimiento de análisis, junto a las fuentes de datos utilizadas. En el apartado de resultados se expone, en primer lugar, el escenario previo a las intervenciones, a continuación, se analiza el escenario actual y, por último, se efectúa una comparación entre ambos. En esta comparación se reflejan las diferencias en cuanto a asentamientos creados y modificaciones en el poblamiento de cada zona. Para finalizar, se ha agregado un apartado donde se discuten los resultados del trabajo y se presentan sus conclusiones. En este sentido, los cambios en el tipo de hábitat se ponen en relación con los procesos de reforma agraria y los conflictos por la tierra que existen en la región.

\section{Metodología}

\section{1. Área de estudio}

La Mesoregión de Presidente Prudente está ubicada entre los Estados de Paraná y Mato Grosso do Sul (Figura 1). Está compuesta por 54 municipios y tiene una población total de 848 mil habitantes (Instituto Brasileiro de Geografía y Estadística [IBGE], 2010). La distribución de esta población está altamente jerarquizada por la ciudad principal, Presidente Prudente, que tiene alrededor de 200 mil habitantes, el 26\% de la población urbana y el $23 \%$ de la población total de la región. Además, hay cinco ciudades pequeñas con una población de entre 39.000 y 24.000 habitantes; diez municipios que tienen entre 22 mil y 11 mil habitantes; y treinta y siete centros con menos de 10.000 habitantes, de los cuales veinticinco tienen menos de 5.000, y siete tienen una población menor a dos mil habitantes. 
Figura 1. Ubicación de la Mesoregión de Presidente Prudente

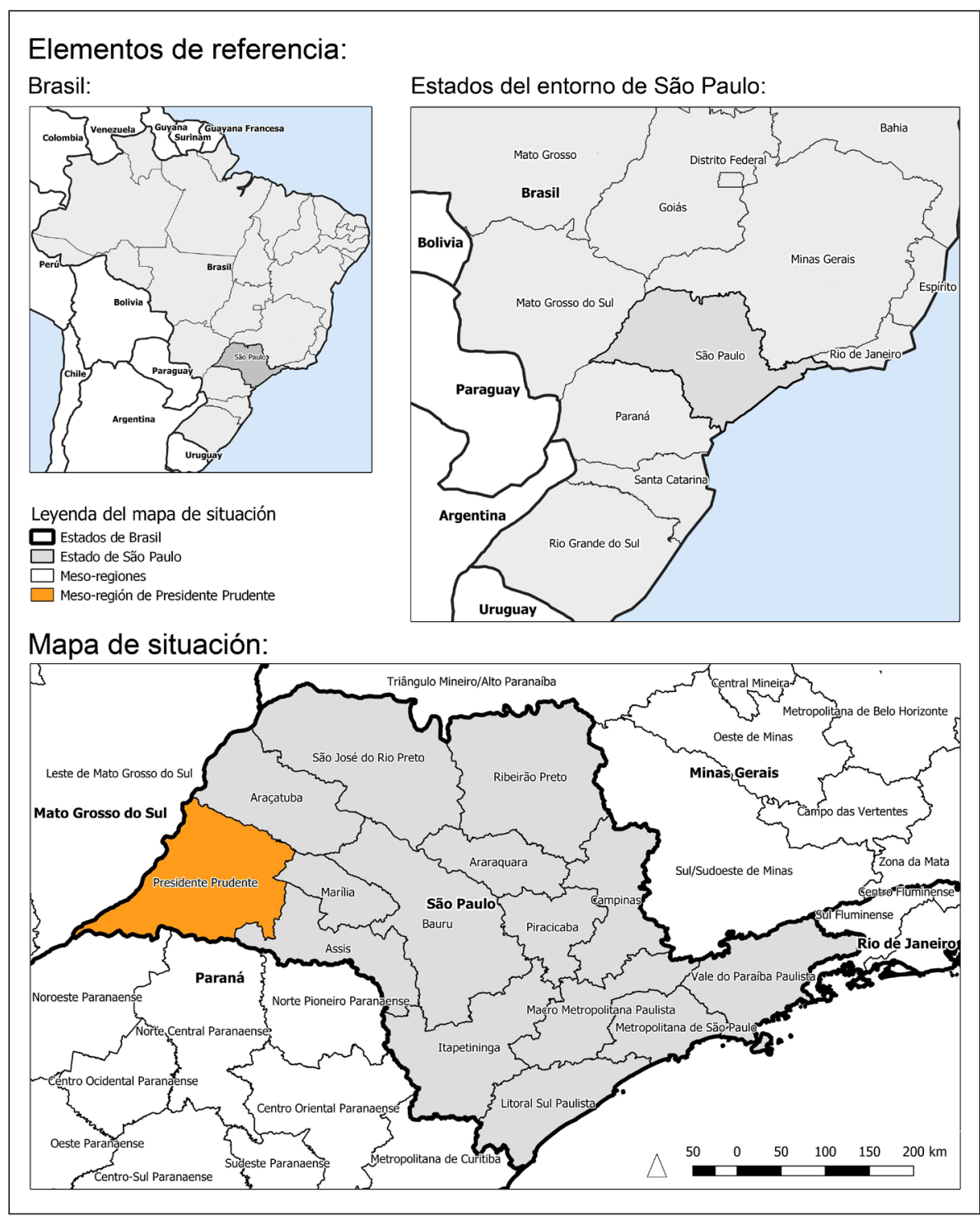

Fuente: Base cartográfica del IBGE (2015). Elaboración propia

El proceso de ocupación en la zona de estudio es relativamente reciente; comenzó a finales del siglo XIX y ha estado profundamente marcado por prácticas de acaparamiento de tierras: prácticamente todas las áreas que componen la región han sido objeto de documentos falsificados destinados a obtener ganancias por su venta o arrendamiento a campesinos, colonos extranjeros o migrantes internos; también para la creación de subdivisiones urbanas y la fundación de aldeas. En la actualidad, la región se compone principalmente de tierras vacantes, es decir, tierras públicas frecuentemente ocupadas de manera irregular; además cuenta con una gran cantidad de trabajadores sin tierra (Leite, 1998).

En la zona, durante la década de 1980 surgieron conflictos serios respecto a las propiedades con títulos controvertidos. La disputa se desarrolló en el ámbito legal y el político, pero también en el de la confrontación directa. En este último, muchas propiedades fueron atacadas por trabajadores sin tierra y por fuerzas policiales ejecutando órdenes de expropiación. Como hito de estas acciones se produjo la ocupación de tierras XV de Novembro, llevada a cabo en 1984. Esta ocupación fue una de las primeras de Brasil y se convirtió en el primer asentamiento de la región, dando acceso a la tierra a 571 familias (Mazzini, 2007).

En la década de 1990, la actividad del MST en la región le hizo ganar notoriedad en todo el país, fundamentalmente por su forma de acción más conocida: la creación de campamentos sobre terrenos 
ocupados. Posteriormente, en estos lugares se asentaba población para trabajar la tierra ocupada. Cuando estos campamentos eran desalojados por la policía, la práctica era trasladar las tiendas de campaña a los bordes de las carreteras. Estas acciones llamaron la atención de la sociedad, llegando a identificar con el MST la presencia de chozas de lona negra, pues este es el color de las tiendas de los campamentos (Fernandes, 1994; Mazzini, 2007).

Ante la presión social, el Estado comenzó a llevar a cabo expropiaciones para asentar familias sin tierra, pagando una indemnización a los propietarios. En el área de estudio, entre 1984 y 2008, se crearon 103 asentamientos rurales distribuidos en 13 municipios. Sin embargo, teniendo en cuenta el grado de concentración de las tierras en la zona, el número es relativamente bajo (Mazzini, 2007). Pese a esto, más de 5.000 familias se han asentado en nuevos núcleos durante estos años (Mazzini, 2007; Mazzini, Martin y Fernandes, 2007; Souza, 2007). La creación de asentamientos por parte del Estado ha tenido efectos positivos en la producción agrícola, en el pequeño comercio y en las condiciones de vida de la población. En los nuevos asentamientos se ofrece a los colonos los servicios básicos de salud y educación, así como la sociabilidad en entornos de ocio y religiosos (Mazzini et al, 2007; Souza, 2007); pese a ello no han cesado las reivindicaciones del MST en la zona (Mazzini, 2007).

\subsection{Escenarios de poblamiento}

La metodología adoptada para valorar el impacto de las políticas de reforma agraria es la comparación de dos escenarios que se han sucedido en el tiempo: en el primero se analiza la distribución de asentamientos y el tipo de hábitat tomando como base las entidades de población existentes con anterioridad a las reformas; en el segundo escenario, se analiza el poblamiento actual considerando los nuevos núcleos de población.

Las políticas de reforma agraria, relacionadas con la creación de nuevos asentamientos rurales, incrementan el número de núcleos de población en el área donde se lleva a cabo la intervención. Teniendo esto en cuenta, desde mediados de la década de 1980 hasta la actualidad, la creación de asentamientos por parte del Estado ha podido introducir importantes cambios en el poblamiento de la región. Para analizar si ha habido cambios en el mismo y, en caso afirmativo, identificar cuáles han sido, se ha planteado el análisis comparativo de dos escenarios. El primero está referido a comienzos de la década de 1990; el segundo refleja la situación actual.

El análisis efectuado ha consistido en comparar la distribución del número de asentamientos en cada escenario para identificar aquellas áreas dónde se ha creado un mayor número de ellos. Adicionalmente se ha analizado el efecto de estas políticas públicas en relación con cambios del tipo de hábitat, disperso o concentrado. En ambos escenarios se ha trabajado a nivel municipal.

\subsubsection{Elementos del sistema de asentamientos}

En la zona de estudio existen núcleos de población de diferentes tipologías: núcleos urbanos (capitales de municipios y villas), barrios rurales, asentamientos rurales y penitenciarías. La capital del municipio, también llamada ciudad o sede, se corresponde con el núcleo dónde se ubica el ayuntamiento. Las villas son entidades de población que, sin ser sede del municipio, son consideradas núcleos urbanos por la ley municipal de Brasil; los barrios rurales son entidades de población dispersa servidas por un camino que pueden estar compuestos por varios diseminados, fundamentalmente se trata de unidades de producción agrícola (Azevedo, 1970). Los asentamientos rurales son conjuntos de unidades agrícolas instaladas por el gobierno brasileño en antiguos latifundios (Instituto de Terras do Estado de São Paulo [ITESP], 2019); por último, las penitenciarías son las prisiones que el gobierno ha radicado en algunos de los municipios que se han analizado.

En el primer escenario anterior a la intervención del gobierno, se incluyen las entidades existentes con anterioridad a 1990, a este respecto se han considerado la capital del municipio, los núcleos urbanos y los barrios rurales. En el segundo escenario, a las entidades de población anteriores, se han añadido los asentamientos rurales y las penitenciarías, pues se trata de entidades que derivan de políticas públicas.

\subsubsection{Tipo de hábitat}

Para facilitar la diferenciación entre hábitat disperso y concentrado, Demangeon (1927) planteó un índice matemático de acuerdo con la información que aparece en los censos de Francia; en ellos "se dan 
dos cifras para el volumen de población del municipio: una para el lugar principal y otra para los écarts, o población dispersa, lo que en el Nomenclator español se denomina entidades" (Canto, Gutierrez y Pérez, 1988, p. 220). A este respecto una entidad singular de población es "cualquier área habitable del término municipal, habitada o excepcionalmente deshabitada, claramente diferenciada dentro del mismo, y que es conocida por una denominación específica que la identifica sin posibilidad de confusión." (Instituto Nacional de Estadística [INE], 2020, on-line).

El coeficiente de dispersión de A. Demangeon $(K)$ lo que hace es relacionar la población que vive en disperso en cada municipio $(E)$ con el número de entidades dispersas $(N)$ y la población total del término municipal o ámbito estudiado $(T)$; su expresión es la siguiente:

$$
K=\frac{E \cdot N}{T}
$$

A los efectos del cálculo se considera población en disperso a aquella que no reside en el núcleo principal del municipio y, del mismo modo, entidades dispersas son todas las existentes salvo la principal; la entidad con mayor volumen de población de todas las del término es considerada la entidad principal. El coeficiente adopta valores más altos cuanto mayor es el grado de dispersión del poblamiento y viceversa (García, Tullas y Perdices, 1995; Zárate y Rubio, 2010). Es decir, Demangeon en esta formulación relaciona mayor dispersión del hábitat con un mayor número de asentamientos dispersos y mayor contingente de población fuera del núcleo principal en relación con la población total del municipio analizado. Los valores más bajos indican un mayor grado de concentración del hábitat; correspondiéndose el 0 con la ausencia de población dispersa o entidades pertenecientes a esta categoría, es decir, con un hábitat totalmente concentrado.

El índice de Demangeon ha sido frecuentemente utilizado para efectuar comparaciones respecto a tipo de hábitat entre diferentes espacios (Canto et al., 1988). Sin embargo, los índices utilizados para analizar el poblamiento también permiten analizar la evolución de una misma zona a lo largo del tiempo (Frutos, Castro y Diago, 1994). En este trabajo, se ha adoptado la formulación de Demangeon para disponer de una descripción cuantitativa del tipo de hábitat de cada municipio en cada escenario. Posteriormente, para identificar los cambios experimentados, el valor del coeficiente de dispersión del escenario previo a la intervención del gobierno se ha comparado con el valor obtenido a partir de la actual distribución de asentamientos. Para conocer la distribución territorial de las variaciones en el índice, los resultados del análisis se han cartografiado, al igual que los valores del coeficiente en cada escenario.

\subsection{Fuentes de información}

La fuente principal para la obtención de datos sobre la distribución de los núcleos de población por municipio ha sido el Censo del IBGE de 2010 (IBGE, 2010a); utilizando datos del IBGE se ha identificado el número total de centros urbanos; sin embargo, esta fuente de datos no proporciona información desglosada sobre la distribución de la población rural. Para solucionar este problema, utilizando fuentes de información adicionales, se ha contabilizado el número de barrios, asentamientos rurales y centros penitenciarios. Los barrios rurales han sido identificados a partir de su toponimia en los mapas municipales del IBGE a escala 1:50.000 ; los datos sobre asentamientos rurales se han tomado del Instituto de Tierras de São Paulo (ITESP) ${ }^{5}$; y los de unidades penitenciarias de la Administración Penitenciaria del Estado de São Paulo (SAP, 2019). Los núcleos urbanos y los barrios rurales preexisten a las políticas públicas y se asocian al escenario de ausencia de intervención del gobierno; el resto de las entidades derivan de la acción reciente del Estado y, junto a las anteriores, se analizan en el escenario de poblamiento actual.

Además de la cuantificación de los núcleos de población de cada municipio, para calcular el coeficiente de dispersión del hábitat se han utilizado datos del censo de Brasil de 1991 y el último disponible, 2010 (IBGE, 2010a) en cuanto a población residente total y población residente en el núcleo principal de cada municipio; de forma adicional a los anteriores, también se ha utilizado el dato de población del censo de

\footnotetext{
4 Recuperado de ftp://geoftp.ibge.gov.br/cartas_e_mapas/mapas_para_fins_de_levantamentos_estatisticos/censo_demografico_2010/mapas_ municipais_estatisticos/sp/

5 Recuperado de http://201.55.33.20/?page_id=3497
} 
2000. Por último, para la elaboración de la cartografía temática de los resultados se ha utilizado la base cartográfica del IBGE (IBGE, 2010b).

\section{Resultados}

\subsection{Tipo de hábitat previo a la intervención del gobierno}

En la región, la mayor parte de los centros de población se crearon antes de 1950. Entre finales del siglo XIX y la primera mitad del XX, la zona atrajo población. Esto se debió al auge de su agricultura de exportación. El primer escenario (Figura 2) refleja la estructura territorial derivada de este proceso: en él se incluyen 81 entidades entre sedes y núcleos urbanos; además, 486 barrios rurales.

Figura 2. Distribución del poblamiento antes de la intervención

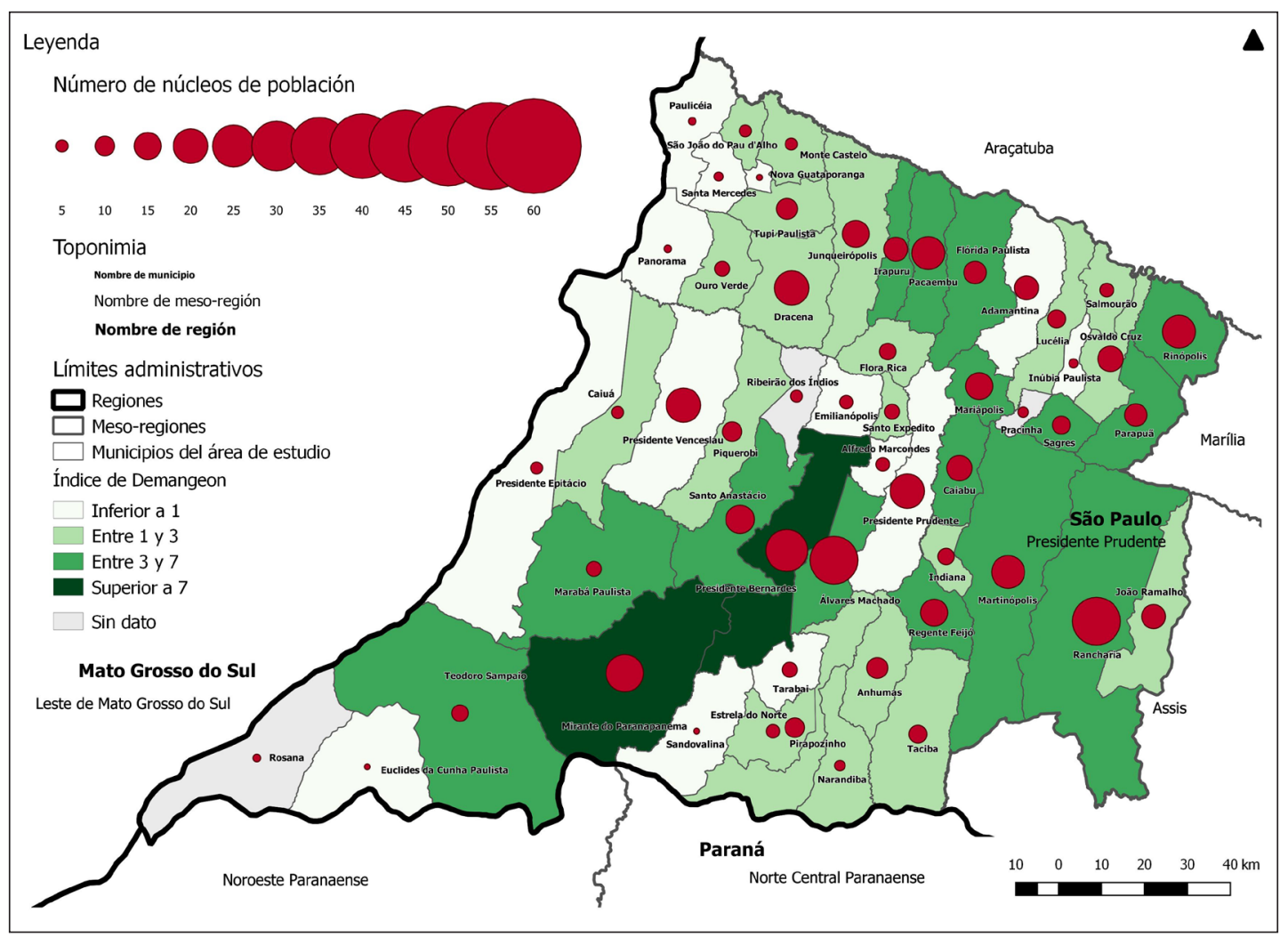

Elaboración propia

La red urbana en el área de estudio está fuertemente jerarquizada en torno a la ciudad de Presidente Prudente: es la capital y en 1990 contaba con el 21,03\% de la población de la región. En la ciudad el grado de concentración del hábitat es muy elevado. En el resto del territorio cabe diferenciar, por un lado, entre la zona norte y sudeste y, por otro, los municipios ubicados al suroeste. En los municipios del norte (tales como Dracena, Adamantina, Junqueirópolis) y el sudeste (Álvares Machado, Alfredo Marcondes, Martinópolis), se instalaron pequeñas propiedades rurales; esto favoreció la aparición de ciudades pequeñas y núcleos secundarios para explotar el territorio.

En cambio, en el suroeste se establecieron explotaciones ganaderas con los centros de población más distantes entre sí. En este contexto se formaron por un lado unos municipios con tendencia a concentrar la población en la sede (Euclides da Cunha Paulista, Presidente Epitácio, Rosana), y, otros, con una mayor dispersión en varios barrios rurales (Teodoro Sampaio, Piquerobi, Mirante do Paranapanema). En estos últimos, los residentes no eran dueños de la tierra, actuando principalmente como trabajadores temporales en las granjas. Como destacan Fernandes y Ramalho (2001), los municipios con más conflictos por la tierra son: Mirante do Paranapanema, Caiuá, Euclides da Cunha Paulista, Teodoro Sampaio, Presidente Epitácio. Es decir, los conflictos son más intensos en las áreas próximas a las fronteras del Estado de São Paulo con los estados de Mato Grosso do Sul y Paraná, en torno a los ríos Paraná y Paranapanema. 


\subsection{Distribución de tipo de hábitat actual}

En el escenario del poblamiento actual, a las 567 entidades preexistentes hay que añadir 103 asentamientos rurales y 24 unidades penitenciarias (Tabla 1). Esto supone un incremento del $22,4 \%$ en el número de entidades. Fundamentalmente se trata de asentamientos rurales.

Tabla 1. Frecuencia de cada tipo de entidad en la actualidad

\begin{tabular}{|c|c|}
\hline Tipo de entidad & Número \\
\hline Sedes y núcleos urbanos & 81 \\
\hline Barrios rurales & 486 \\
\hline Asentamientos rurales & 103 \\
\hline Penitenciarías & 24 \\
\hline Total & 694 \\
\hline
\end{tabular}

Fuente: Censo demográfico do IBGE (2010a); Base Cartográfica do IBGE (2010b); ITESP (2019); Secretaria de Administração Prisional (2020). Elaboración propia

En la actualidad se mantiene un alto grado de concentración en la capital; así mismo, en el resto de las zonas, los municipios ubicados a norte y sureste mantienen valores similares a los previamente observados tanto en cuando a núcleos de población como a dispersión del poblamiento (Figura 3). Sin embargo, a día de hoy, los municipios con mayor número de núcleos de población se encuentran en el suroeste de la región; destacan con 3 o más asentamientos rurales: Mirante do Paranapanema, Teodoro Sampaio, Presidente Bernardes, Euclides da Cunha Paulista, Marabá Paulista, Presidente Venceslau, Caiuá y Rosana. Esta misma zona destaca por ser aquella con una dispersión del poblamiento mayor.

Figura 3. Distribución actual del poblamiento

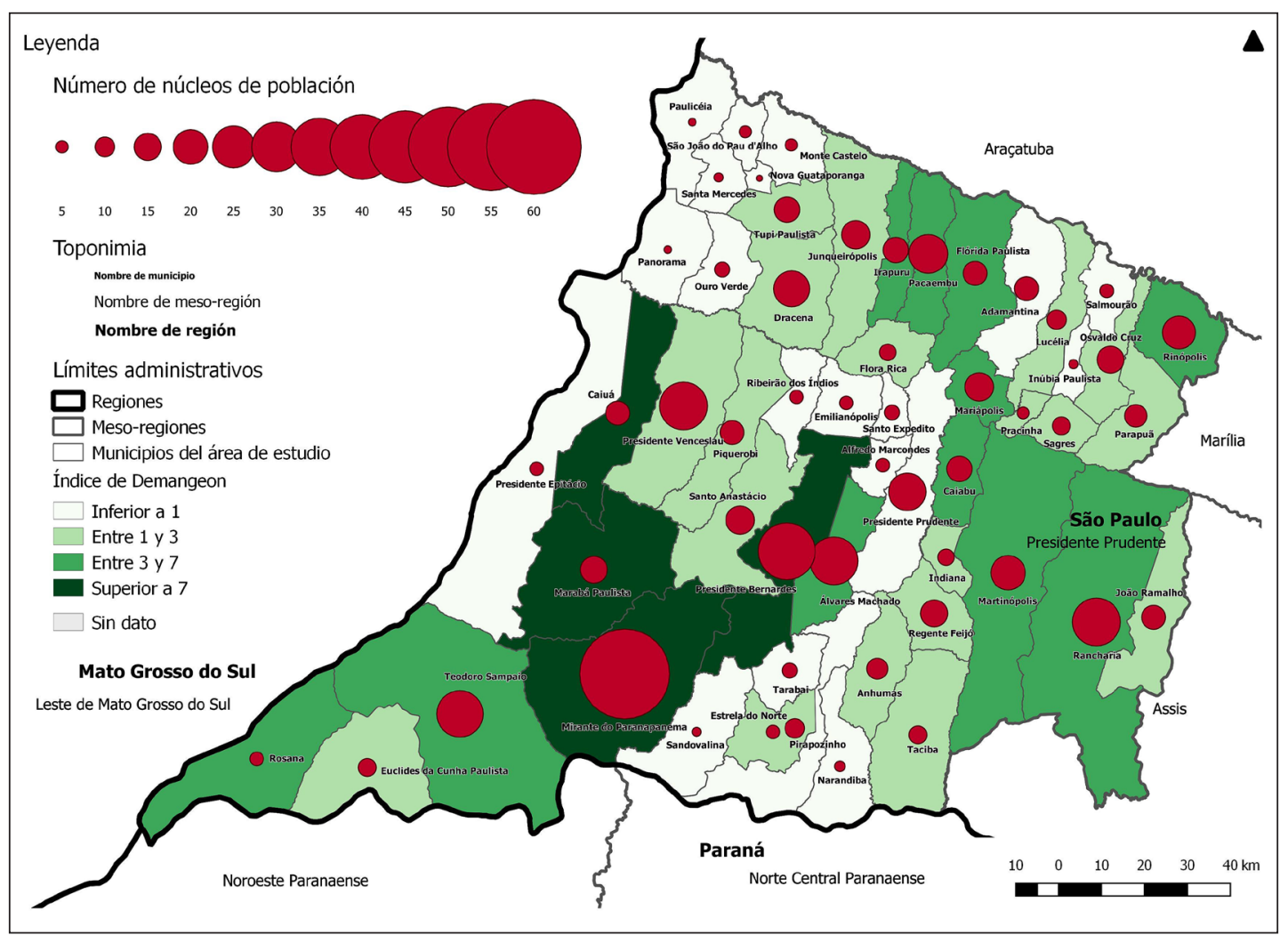

Elaboración propia

En la región hay un total de 24 centros penitenciarios, instalados principalmente entre 1990 y la década de 2000; su población puede alcanzar los 1.500 reclusos. Sin embargo, a diferencia de los asenta- 
mientos rurales, que se concentran en la zona del suroeste, estos se encuentran repartidos por todo el territorio: existen 2 o más penitenciarías en los municipios de Pacaembu, Presidente Bernardes, Presidente Venceslau, Caiuá, Tupi Paulista y Presidente Prudente.

\subsection{Análisis de cambios}

Al comparar ambos escenarios (Figura 4), se pone de relieve que no todos los municipios de la región han experimentado una evolución similar entre 1990 y 2010 en lo que respecta al poblamiento: de los 51 municipios para los que se cuenta con información referida a 1991 y 2010, en 25 ha aumentado la concentración, en 12 no ha habido cambios y en 14 ha aumentado la dispersión.

Figura 4. Distribución territorial de los cambios en el poblamiento entre 1991 y 2010

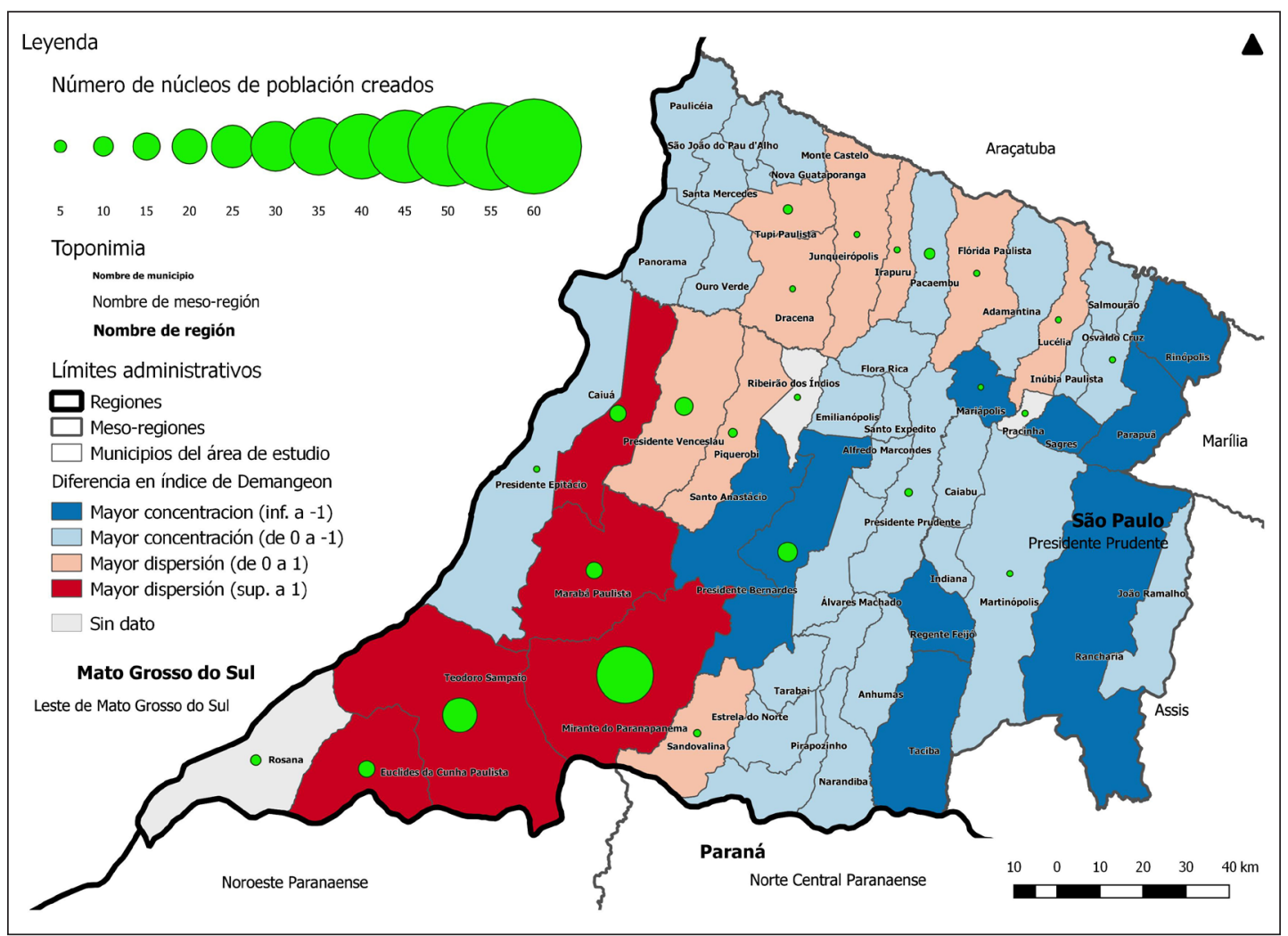

Elaboración propia

Los municipios del suroeste, que son los que tradicionalmente han mantenido mayores conflictos sobre las tierras (Fernandes y Ramalho, 2001), son aquellos en los que la política de creación de asentamientos ha tenido mayor impacto. Mientras el resto del territorio se ha mantenido estable, en esta zona se aprecia un incremento del número de entidades y un incremento del grado de dispersión del poblamiento. Frente a estos, el hábitat de otros municipios ha aumentado su grado de concentración. A este respecto cabe mencionar el ejemplo de Rancharia: este municipio cuenta con 26 barrios rurales; pese a esto, en él ha aumentado el grado de concentración debido a un incremento del tamaño de la sede que ha pasado de 22.236 habitantes de 1991 a 24.931 en 2010. En otros municipios como el de la capital de la región, Presidente Prudente, el número de habitantes en la sede se ha incrementado en 42.452 personas, pasando de 157.680 a 200.132 entre 1991 y 2010.

En la zona suroeste, donde se ha creado un mayor número de entidades, destacan los municipios de Mirante do Paranapanema y Teodoro Sampaio; en ellos se han instalado 35 y 20 asentamientos rurales respectivamente. Mirante do Paranapanema tiene un área de $1.239 \mathrm{~km}^{2}$ y 19 barrios rurales, entre 1991 y 2010 ha pasado de tener 15.179 habitantes a contar con 17.059, pese a ello la población residente en la sede se ha reducido: de 7.814 a 7.568 ; frente a esto, la población residiendo fuera de la sede se ha incrementado en 2.126 personas, esto supone un $10,48 \%$. La importancia de este dato se pone de manifiesto 
en relación con la evolución de la población viviendo fuera de la sede del municipio en el conjunto de la región, que ha sido de un $-27,61 \%$ (Tabla 2). Por su parte, en Teodoro Sampaio la población total pasó de 49.236 habitantes en 1991 a 20.003 en 2000 por un proceso de segregación en su límite municipal en los años 90. Este proceso culminó en la creación de los municipios de Rosana e Euclides da Cunha, por la ley del Estado de São Paulo n ${ }^{\circ}$ 6645, de 9 de enero de 1990; por tanto, para analizar este caso es preferible utilizar como base el año 2000. Teodoro Sampaio tiene un área de $1.556 \mathrm{~km}^{2}$ y 6 barrios rurales; sin embargo, pese a la creación de asentamientos la población residiendo fuera de la sede se ha reducido en un $2,08 \%$ frente a un incremento del $13,68 \%$ del núcleo principal.

Tabla 2. Distribución de la población en la Mesoregión de Presidente Prudente por lugar de residencia entre los años 1991 y 2010

\begin{tabular}{|l|r|r|r|r|r|r|}
\hline \multirow{2}{*}{ Lugar de residencia } & \multicolumn{2}{|c|}{ Población en 1991 } & \multicolumn{2}{c|}{ Población en 2010} & \multicolumn{2}{c|}{ Diferencia entre 2010 y 1991} \\
\cline { 2 - 7 } & $\begin{array}{c}\text { Número } \\
\text { de habitantes }\end{array}$ & Porcentaje & $\begin{array}{c}\text { Número } \\
\text { de habitantes }\end{array}$ & Porcentaje & $\begin{array}{c}\text { Número } \\
\text { de habitantes }\end{array}$ & $\begin{array}{c}\text { Porcentaje } \\
\text { sobre 1991 }\end{array}$ \\
\hline Sede del municipio & 593.970 & 79,20 & 735.218 & 86,69 & 141.248 & 23,78 \\
\hline Resto del término & 155.972 & 20,80 & 112.906 & 13,31 & -43.066 & $-27,61$ \\
\hline Población total & 749.942 & 100,00 & 848.124 & 100,00 & 98.182 & 13,09 \\
\hline
\end{tabular}

Fuente: Censo demográfico del IBGE (1991; 2010a). Elaboración propia

El incremento de la dispersión es muy llamativo en los municipios donde se han creado los nuevos asentamientos. La tendencia al incremento del tamaño de las sedes que se observa en el conjunto de la región únicamente se ha contrarrestado en Presidente Bernardes, Presidente Epitácio y Ribeirão dos Índios (Tabla 3). Frente a estos, en la mayor parte de los términos donde se han creado asentamientos ha aumentado la dispersión, aunque la población de su sede haya crecido. Este hecho se observa en municipios como Teodoro Sampaio, Rosana, Sandovalina, Tupi Paulista, Presidente Venceslau y Piquerobi. Por otro lado, pese a la tendencia generalizada al aumento de población en las sedes de los municipios de la zona de estudio, en algunos de los municipios donde la creación de asentamientos ha sido más intensa el porcentaje de población dispersa se ha incrementado entre 1991 y 2010. Esto ha ocurrido en: Mirante do Paranapanema, Caiuá y Marabá Paulista.

Tabla 3. Municipios en los que se crean asentamientos rurales: evolución del tipo de hábitat, población en la sede y número de asentamientos

\begin{tabular}{|c|c|c|c|c|}
\hline \multirow{3}{*}{ Municipio } & \multicolumn{3}{|c|}{ Diferencia 2010-1991 } & \multirow{3}{*}{$\begin{array}{l}\text { Número de } \\
\text { asentamientos } \\
\text { (2010) }\end{array}$} \\
\hline & \multirow{2}{*}{$\begin{array}{l}\text { Evolución } \\
\text { del índice de } \\
\text { Demangeon }\end{array}$} & \multicolumn{2}{|c|}{ Evolución de la población de la sede } & \\
\hline & & $\begin{array}{c}\text { Número } \\
\text { de habitantes }\end{array}$ & $\begin{array}{c}\text { Porcentaje sobre el } \\
\text { municipio }\end{array}$ & \\
\hline Mirante do Paranapanema & 20,97 & -246 & $-7,12$ & 35 \\
\hline Caiuá & 4,94 & 470 & $-5,4$ & 6 \\
\hline Teodoro Sampaio* & 4,61 & 1.491 & 2,18 & 20 \\
\hline Marabá Paulista & 4,44 & 238 & $-9,98$ & 7 \\
\hline Euclides da Cunha Paulista* & 2,9 & -320 & 0,79 & 8 \\
\hline Rosana* & 2,84 & 9.660 & 54,95 & 4 \\
\hline Sandovalina & 0,6 & 936 & 1,32 & 2 \\
\hline Tupi Paulista & 0,36 & 577 & 2,86 & 1 \\
\hline Presidente Venceslau & 0,3 & 1.884 & 0,47 & 7 \\
\hline Piquerobi & 0,29 & 365 & 4,96 & 3 \\
\hline Ribeirão dos Índios* & $-0,06$ & 90 & 5,38 & 1 \\
\hline Presidente Epitácio & $-0,1$ & 7.566 & 22,53 & 1 \\
\hline Presidente Bernardes & $-2,39$ & 1.727 & 4,93 & 8 \\
\hline
\end{tabular}

*Sin información completa de 1991; se muestran diferencias entre 2010 y 2000.

Fuente datos de Censo demográfico del IBGE (2010a); Bases Cartográficas del IBGE (2015); ITESP (2019); Secretaria de Administração Prisional (2020). Elaboración propia 


\section{Discusión de resultados}

\subsection{Efecto de las reformas en el hábitat rural}

En este trabajo se ha utilizado el índice de Demangeon, un índice clásico para analizar el poblamiento, como herramienta para analizar los cambios en el tipo de hábitat en relación con las políticas de reforma agraria puestas en marcha en la Mesoregión de Presidente Prudente (São Paulo). En los resultados se observa que, en la práctica totalidad de los términos en los que se han creado asentamientos rurales, la dispersión ha aumentado. Las zonas donde el número de asentamientos es mayor coinciden en lo esencial con las identificadas por Fernandes y Ramalho (2001) como zonas con graves problemas sobre titularidad de las tierras. Entre ellas destacan: Mirante do Paranapanema, Euclides da Cunha Paulista, Teodoro Sampaio y Presidente Epitácio. Es decir, el área suroeste de la región, próxima a la frontera con Mato Grosso del Sur y Paraná.

La creación de asentamientos ha sido capaz de contrarrestar la tendencia a la concentración asociada al aumento del número de habitantes que se observa en las sedes. Los asentamientos rurales han producido un aumento de la población en el campo (Souza, 2007). En cambio, las zonas donde el poblamiento es más antiguo, ubicadas fundamentalmente al este de la región, presentan un hábitat más concentrado en 2010 que en 1991. Esto pone de relieve el papel de las reformas a la hora de introducir cambios en la organización territorial de los lugares donde se llevan a cabo.

En otras zonas del país Heredia, Medeiros, Palmeira, Cintrão y Leite (2002) también observaron, asociada a la creación de asentamientos rurales, una modificación en el patrón de la distribución de la población. Al respecto pusieron de manifiesto que estas políticas provocan un rediseño real de las áreas rurales que va desde la apertura de nuevos caminos, la revitalización de los medios de comercialización y el fortalecimiento de los lazos entre el mundo rural y el urbano. Sin embargo, la política de creación de asentamientos no solo tiene efectos en el hábitat y el modelo productivo; también los tiene en otros aspectos tales como son el fomento de la autonomía de los distritos y la creación de nuevos municipios (Heredia et al., 2002).

Los resultados confirman que la dinámica agraria, específicamente la relacionada con la estructura de la tierra, incide sobre la forma de ocupar el territorio. En el caso de las reformas puestas en marcha en la región, la tendencia ha sido hacia una mayor dispersión. Esto contrasta con otros modelos económicos como el hábitat tradicional de las plantaciones, que era concentrado. En lo que respecta esto "los factores de agrupamiento de la población agrícola son o bien factores antiguos, procedentes de condiciones desaparecidas, pero que han engendrado formas capaces de sobrevivirles, o bien factores recientes." (George, 1974, p. 187). En este sentido, la tendencia a la dispersión que se ha observado en el suroeste de la región es un factor reciente, derivado directamente de una intervención pública orientada a modificar las estructuras agrarias y que ha tenido como efecto una mayor dispersión del hábitat.

El análisis del poblamiento se puede complementar con un estudio sobre posibles cambios en el patrón de distribución espacial de los núcleos de población dentro de cada municipio; también, con un análisis comparativo entre los patrones que se observan en las zonas de reforma situadas al suroeste de la Mesorregión de Presidente Prudente y las del este, donde el poblamiento es más antiguo. Para ello, al igual que se ha hecho en este trabajo, es posible realizar análisis diacrónicos utilizando índices clásicos. En este caso, Racionero (1986) planteaba la utilización del índice de Clark-Evans para analizar la realidad del sistema de asentamientos desde el punto de vista de la regularidad espacial de su distribución.

En la Mesoregión de Presidente Prudente se ha contabilizado un total de 103 nuevos asentamientos rurales entre los dos escenarios analizados. Su creación se ha concentrado en áreas con alta conflictividad y presencia de movimientos como el MST; sin embargo, estos últimos no han cesado en sus demandas (Mazzini, 2007). Los asentamientos han tenido un efecto positivo en la calidad de vida de la población (Mazzini et al., 2007) y han contribuido a diversificar la economía (Souza, 2007). Los efectos positivos de este tipo de reformas pueden convertir la creación de asentamientos en un instrumento útil para la ordenación del territorio y las políticas de desarrollo regional (Mazzini, 2007). Teniendo esto en cuenta, con el objetivo de evitar posibles problemas derivados de su implantación, puede ser interesante complementar los estudios acerca de aspectos como la calidad de las tierras con otros como su grado de integración en la red urbana, accesibilidad a los mercados y disponibilidad de servicios. Ante un aumento de la dispersión del hábitat, es un aspecto clave asegurar a los nuevos núcleos una accesibilidad suficiente como para garantizar que el acceso a los servicios públicos se lleva a cabo dentro de unos tiempos de desplazamiento razonables. 
En relación con los servicios y el incremento de la dispersión del hábitat rural, cabe plantearse como es la relación funcional que estos nuevos asentamientos mantienen con las sedes de los municipios. Es decir, si se mantiene un esquema funcional clásico en el que la sede actúa como cabecera y centro de abastecimiento, mientras los asentamientos constituyen lugares de producción dispersos que cuentan con una centralidad baja y un número reducido de funciones. En este sentido, cabría plantear análisis acerca de la distribución espacial de las actividades económicas que se llevan a cabo y de los servicios que prestan. En los modelos urbanos colonialistas "las ciudades están localizadas de manera que sirven de centros de recolección y explotación de materias primas" (Racionero, 1986, p. 152). Sin embargo, para asegurar la calidad de vida de la población que reside en estos lugares es necesario disponer de determinados servicios públicos esenciales.

\subsection{Los asentamientos rurales en la ordenación del territorio}

Brasil es un país con algunos problemas sociales de carácter estructural; entre ellos, aunque tiene grandes extensiones de terreno prácticamente deshabitadas, destaca el desigual reparto de la tierra y la riqueza. En los últimos años se han emprendido políticas de reforma agraria para paliarlo (Peixoto, 2017). El papel del Estado ha sido determinante a la hora de crear nuevos asentamientos que sirvan como base para poder explotar el territorio y aprovechar sus recursos. Sin embargo, en este proceso es fundamental el respeto por el medio ambiente y la sostenibilidad de tal explotación. En el contexto de las políticas de desarrollo territorial, vale la pena recordar que "el método clásico para desarrollar una región rica en recursos naturales y no ocupada es realizar prospecciones y asentar junto a las materias primas halladas las poblaciones necesarias para su extracción" (Racionero, 1986, p. 152). En relación con esta cuestión, los asentamientos rurales son un elemento a considerar dentro de estas políticas de desarrollo (Mazzini et al., 2007).

El potencial agrícola de Presidente Prudente es muy alto; sin embargo, los problemas y conflictos existentes en el campo han impedido su óptimo aprovechamiento (Dundes, 2007). Desde la década de 1970, con estímulo del Estado y en un contexto internacional marcado por la crisis del petróleo, y, más fuertemente, desde la década de 2000, con la llegada de los automóviles de combustible flexible (que funcionan con gasolina o etanol), se han instalado en la región algunas plantas industriales de azúcar y alcohol. En este sentido, se ha adoptado el cultivo de la caña de azúcar con plantaciones en grandes propiedades para reemplazar el ganado de carne (Segatti, 2009). Sin embargo, este sistema no es el único en el que este cultivo se desarrolla; también es frecuente la formación de plantaciones mediante el arrendamiento de múltiples propiedades de pequeño tamaño. Esto se ha visto favorecido por el hecho de que muchos pequeños propietarios sean de edad avanzada y que sus hijos no hayan continuado la actividad agraria sino otras desarrolladas en ámbitos urbanos. Así mismo, existen algunos beneficiarios de políticas de reforma agraria que también arriendan sus tierras para las empresas de caña de azúcar. Gonçalves (2009), apunta que tal práctica tiene diferentes motivaciones: por un lado, es ventajoso para las empresas que así no han de preocuparse por la compra de las tierras y, a la par, se benefician indirectamente de áreas y créditos públicos. Por otro lado, es ventajoso para los asentados que se aseguran un ingreso periódico. Esta práctica es problemática, pues además de suponer una desviación de los objetivos perseguidos por la reforma agraria (Gonçalves, 2009; Menezes, Mallmann, Aragão, Fernandes y Fernandes, 2018), en ocasiones se producen atrasos e incluso faltas de pago a los arrendadores y, al finalizar los contratos, es frecuente el empobrecimiento del suelo (Menezes et al, 2018; Segatti, 2009).

En un primer momento, las plantaciones de caña de azúcar generaron nuevos empleos. Sin embargo, el trabajo de cortar caña de azúcar es considerado un trabajo mal pagado, duro y poco saludable; como plantean Messias y Lizarazo (2019), se relaciona con Lesiones por Esfuerzo Repetitivo (LER) y con Trastornos Músculo-Esqueléticos (TME) de origen laboral. Además de esto, la práctica de quemar paja, que se realiza con el corte manual, está considerada como algo perjudicial para el medio ambiente. Por estas razones, actualmente el corte se realiza con maquinaria, quedando limitada su práctica manual a las áreas donde no se puede mecanizar, cambio que ha terminado por producir un aumento del desempleo (Barreto, 2012).

Desde el punto de vista de la industria, la región no ha conseguido atraer a las grandes empresas del sector. Además, en esta actividad, existe una alta vulnerabilidad a las crisis: ante ellas, muchas plantas se declaran en bancarrota, dejando a los trabajadores en paro y a los propietarios sin percibir sus rentas por los arrendamientos. Por tanto, incluso en una región con poca industria, el impacto de las plantas de 
azúcar y alcohol no puede considerarse completamente positivo. A esto se suma el hecho de que se trata de un desarrollo exógeno que no fija sus raíces en la región (Fonseca, 2015). Frente a esto, la agricultura familiar, representada por los campesinos locales y los pequeños propietarios rurales es una opción acorde con los preceptos del desarrollo sostenible, en sus variables humanas y ambientales.

Entre 1950 y 1960 el éxodo rural fue fundamental en el crecimiento de grandes ciudades como São Paulo y Rio de Janeiro. Así mismo, en la Mesoregión de Presidente Prudente existe una tendencia a la concentración de población en las sedes y, sobre todo, en la capital regional, Presidente Prudente; frente a esto, los espacios rurales pierden población año tras año. Esta tendencia puede reproducir, a nivel regional, escenarios negativos similares a los derivados de la concentración de población y la escasez de recursos que se observan en las grandes ciudades; fundamentalmente: desempleo, pobreza, problemas en el acceso a la vivienda, presión sobre los sistemas de transporte y contaminación, aumento de la criminalidad y falta de acceso a servicios públicos esenciales como salud y educación. Muchas de estas cuestiones no son analizadas mediante una visión integral del territorio; sin embargo, podrían contemplarse de forma holística, junto a otros aspectos como los agrarios.

La política de asentamientos rurales puede crear espacios para vivir en el campo, con garantía de trabajo y calidad de vida; esto, además, dado el crecimiento de la tasa de urbanización del país, puede tener aspectos positivos sobre el futuro demográfico del mundo rural (Mattei, 2014). Teniendo en cuenta los desafíos que supone una mayor dispersión del hábitat en cuanto al acceso a los servicios públicos, los efectos positivos sobre la calidad de vida que se han observado en los asentamientos rurales suscitan la posibilidad de utilizar estas medidas en políticas de reducción de la pobreza y desarrollo sostenible; es decir, como una herramienta para la ordenación del territorio que permita mejorar el nivel de vida de la población.

\section{Conclusiones}

Las demandas sociales, a través de instrumentos como la reforma agraria, pueden incidir sobre el territorio. En el caso de estudio analizado, pese a existir movimientos de población del campo a la ciudad, en la mayor parte de los municipios rurales afectados por políticas de creación de asentamientos, la dispersión ha aumentado. Es decir, se confirma que las políticas de reforma agraria pueden ser capaces de modificar el hábitat rural y, por tanto, el poblamiento existente en el territorio donde se llevan a cabo. Estos cambios se han observado en un periodo de tan solo 20 años, lo que pone de manifiesto su celeridad. Ante un aumento de la dispersión, desde el punto de vista de la Ordenación del Territorio, se debe señalar la importancia de los análisis sobre la accesibilidad a servicios públicos de las nuevas comunidades.

Respecto a la metodología, el estudio comparativo de escenarios utilizando índices clásicos de la Geografía puede aportar una renovada utilidad a estos últimos. Su aplicación en estudios diacrónicos, más allá de la descripción territorial de categorías como las de hábitat disperso o concentrado, puede permitir identificar procesos que afectan al territorio y delimitar las áreas que están siendo afectadas por ellos. En este sentido, en este trabajo ha sido posible relacionar problemas y demandas sociales, ejecución de políticas de reforma y cambios en el poblamiento a través del coeficiente de Demangeon, formulado en el año 1927. Esto, pone de relieve la utilidad de esta metodología y, también, que las demandas sociales, a través de políticas públicas, pueden repercutir sobre el modo en que se ocupa y organiza el espacio geográfico.

\section{Referencias}

Aguilera, M.J., Borderías, M.P., González, M.P. y Santos, J.M. (1991). Geografía general II (Geografía Humana). Madrid: Ed. Uned.

Azevedo, A. de (1970). Brasil: A Terra e o Homem. São Paulo: Ed. Nacional/Edusp.

Barreto, M.J. (2012). Territorialização das agroindústrias canavieiras no Pontal do Paranapanema e os desdobramentos para o trabalho (Trabajo de fin de máster). Universidade Estadual Paulista, Faculdade de Ciências e Tecnologia, Programa de Pós-Graduação em Geografia. Recuperado de http://hdl.handle. $\underline{\text { net/11449/96702 }}$

Bernardes, J. y Maldonado, G. (2017). Estratégias do capital na fronteira agrícola moderna brasileira e argentina. En J. Bernardes, S. Frederico, C. Gras, V. Hernandéz, y G. Maldonado (Org.), Globalização 
do agronegócio e land grabbing: A atuação das megaempresas argentinas no Brasil (pp. 57-81). Brasil: Lamparina, Capes, Faperj, Reagri y Ministerio de Ciencia, Tecnologia e Innovación Productiva.

Canto, C., Gutierrez J., y Pérez, M.C. (1988). Asentamientos y transportes. En C. Canto, J. Gutierrez y M.C. Pérez (Autores), Trabajos Prácticos de Geografía Humana (pp. 201-310). Síntesis.

Cosme, C.M. (2016). Reforma agrária no Brasil do século XXI: qual reforma agrária? Boletim DATALUTA, 106, 1-25. Recuperado de http://www2.fct.unesp.br/nera/artigodomes/10artigodomes_2016.pdf

D’Entremont, A. (1997). Geografía Económica. Madrid: Ed. Cátedra.

Demangeon, A. (1927). La géographie de l'habitat rural. Annales de Géographie.

Dundes, A.C. (2007). Região do devir e Região do atraso: discurso e representações sobre a Região de Presidente Prudente-SP (Tesis doctoral). Universidade Estadual Paulista, Faculdade de Ciências e Tecnologia, Programa de Pós-Graduação em Geografia. Brasil. Recuperado de http://www2.fct.unesp. br/pos/geo/dis_teses/07/anaclaudiadundes.pdf

Ebling, L.A. (2009). Apropriação da terra e formação de grandes patrimônios fundiários na fronteira sul do Brasil, através dos inventários post mortem (1800-1860). Trabajos y Comunicaciones, (35), 1-23. Recuperado de https://dialnet.unirioja.es/servlet/articulo?codigo=3862667

Fernandes, B.M. (1994). Espacialização e Territorialização da Luta Pela Terra: A Formação do MST Movimento Dos Trabalhadores Rurais Sem Terra - no Estado de São Paulo (Trabajo de fin de máster). Universidade de São Paulo, Faculdade de Filosofia, Letras e Ciências Humanas. Brasil.

Fernandes, B.M. (2008). O MST e as reformas agrárias do Brasil. Revista Osal, 9, 73-85.

Fernandes, B.M.y Ramalho, C.B. (2001). Luta pela terra e desenvolvimento rural no Pontal do Paranapanema (SP). Estudos avançados, 15(43), 239-254. https://doi.org/10.1590/S0103-40142001000300018

Fonseca, R.O. da (2015). A descentralização político-administrativa no Brasil: um estudo sobre a disputa entre os municípios de Caiuá e Marabá Paulista pela DECASA Açúcar e Álcool a partir das escalas geográficas de análise (Trabajo de fin de grado). Universidade Estadual Paulista, Faculdade de Ciências e Tecnologia. Brasil. Recuperado de https://repositorio.unesp.br/handle/11449/124157

Frutos, L.M., Castro, M.S. y Diago, M.C.C. (1994). Cambios en el sistema de asentamientos rurales: la provincia de Teruel. Geographicalia, (31), 83-94.

García, M.D., Tullas, A.F.T.I. y Perdices, N.V. (1995). Geografía Rural. Síntesis.

George, P. (1974). Geografía rural. Barcelona: Ed. Ariel.

Germani, G.I. (1997). Condiciones históricas sociales que regulan el acceso a la tierra en el espacio agrario de Brasil. Scripta Nova. Revista Electrónica de Geografía y Ciencias Sociales, 6, 1-13.

Girardi, E. P., y Fernandes, B. M. (2008). A luta pela terra e a política de assentamentos rurais no Brasil: a Reforma Agrária conservadora. Agrária (São Paulo. Online), 8, 73-98. https://doi.org/10.11606/ issn.1808-1150.v0i8p73-98

Gonçalves, E. C. (2009). Disputa territorial: a "parceria" entre os assentados e usinas de açúcar e álcool. En IV Simpósio Internacional de Geografia Agrária - V Simpósio Nacional de Geografia Agrária (pp. 757-770). Niterói: Universidade Federal Fluminense. https://dx.doi.org/10.1590/S1517-45222011000100011

Heredia, B., Medeiros, L.S., Palmeira, M., Cintrão, R. y Leite, S. (2002). Análise dos impactos regionais da reforma agrária no Brasil. Estudos Sociedade e Agricultura, 10(1), 73-111. Recuperado de https:// revistaesa.com/ojs/index.php/esa/article/view/209

Ilbery B.W. (1985). Agricultural Geography - A Social and Economic Analysis. Oxford: Oxford University Press.

Instituto Brasileiro de Geografia (IBGE) (1991). Censo demográfico. Recuperado de http://www.sidra.ibge. gov.br

Instituto Brasileiro de Geografia (IBGE) (2000). Censo demográfico. Recuperado de http://www.sidra.ibge. gov.br

Instituto Brasileiro de Geografia (IBGE) (2010a). Censo demográfico. Recuperado de http://www.sidra. ibge.gov.br

Instituto Brasileiro de Geografia (IBGE) (2010b). Base cartográfica. Recuperado de https://mapas.ibge.gov. br/bases-e-referenciais/bases-cartograficas/malhas-digitais 
Instituto Brasileiro de Geografia (IBGE) (2015). Base cartográfica. Recuperado de https://mapas.ibge.gov. br/bases-e-referenciais/bases-cartograficas/malhas-digitais

Instituto de Terras do Estado de São Paulo (ITESP) (2019). Assentamentos Rurais. Fundação Instituto de Terras do Estado de São Paulo. Recuperado de http://201.55.33.20/?Page_Id=3497

Instituto Nacional de Estadística (INE) (2020). Nomenclátor. Relación de unidades poblacionales (Metodología). Recuperado de https://www.ine.es/nomen2/Metodologia.do

Leite, J.F. (1998). A Ocupação Do Pontal Do Paranapanema. São Paulo: Hucitec.

Lima, F. L. S. de, Locatel, C. D. y Silva, C. C. L. da (2012). Modernização seletiva da agricultura: o avanço do agronegócio da soja no sul do maranhão. En Territórios em disputa: os desafios da geografia agrária nas contradições do desenvolvimento brasileiro-XXI Encontro Nacional de Geografia Agrária, Actas (pp. 1-18). Uberlândia: Universidade Federal de Uberlândia.

Márquez, D. (1992). Los sistemas agrarios. Madrid: Ed. Síntesis.

Martins, J. de S (1996). O cativeiro da terra. São Paulo: Hucitec.

Mattei, L. F. (2012). A reforma agrária brasileira: evolução do número de famílias assentadas no período pós-redemocratização do país. Estudos Sociedade e Agricultura, vol. 20, n. 1, 301-325. Recuperado de https://revistaesa.com/ojs/index.php/esa/article/view/356

Mattei, L. (2014). O papel e a importância da agricultura familiar no desenvolvimento rural brasileiro contemporâneo. Revista Econômica do Nordeste, 45(5), 83-92.

Mazzini, E. de J.T. (2007). Assentamentos Rurais no Pontal do Paranapanema - SP: uma política de desenvolvimento regional ou de compensação social? (Trabajo de fin de máster). Universidade Estadual Paulista, Faculdade de Ciências e Tecnologia, Programa de Pós-Graduação em Geografia. Brasil. Recuperado de https://repositorio.unesp.br/handle/11449/96775

Mazzini, E. de J.T., Martin, E.S. y Fernandes, B.M. (2007). Assentamentos Rurais no Pontal do Paranapanema - SP: uma política de desenvolvimento regional. Formação (Online), 1(14), 56-66. https://doi.org/10.33081/formacao.vli14.696

Menezes, L. de F, Mallmann, V., Aragão, L. W. R., Fernandes, S. S. L. y Fernandes, T. C. L. (2018). Porque os assentados da reforma agrária brasileira arrendam suas terras?. Cadernos de Agroecologia, 13(2), 1-6. Recuperado de http://cadernos.aba-agroecologia.org.br/index.php/cadernos/article/view/2177

Messias, I.A. y Lizarazo, R. P. (2019). A vigência do medo, sofrimento e sobrecarga física para o trabalhador no corte da cana de açúcar no estado de São Paulo. Confins, 41, 1-15. https://doi.org/10.4000/ confins. 21313

Molinero, F. (1990). Los espacios rurales. Barcelona: Ed. Ariel.

Navarro, Z. (2017). O mundo rural brasileiro: história, tendências atuais e os desafios principais do "próximo período". Revista de economia e Agronegócio, 15(3), 293-298. https://doi.org/10.25070/rea. v15i3.544

Oliveira, A. U de (2009). Os agrocombustíveis e a produção de alimentos. En Caminando en una América Latina en transformación - XII Encuentro de Geógrafos de América Latina (pp. 1-15). Montevideo: Universidad de Montevideo.

Oliveira, A. U de (2015). Reforma agrária, grilagem das terras públicas e a luta pela terra e território no Brasil. En Por una América Latina unida y sustentable - XV Encuentro de Geógrafos de América Latina (pp. 1-15). La Habana: Universidad de Habana.

Ortega, A. C. y Jesus, C. M. (2011). Território café do Cerrado: transformações na estrutura produtiva e seus impactos sobre o pessoal ocupado. Revista de Economia e Sociologia Rural, 49(3), 771-800. https://doi.org/10.1590/S0103-20032011000300010

Osorio, L.M. (2006). Tierras nuevas y la construcción del estado en Brasil y Argentina. América Latina en la historia económica, (25), 43-71. https://doi.org/10.18232/alhe.v13il.371

Peixoto, S. E. A. A. (2017). A reforma agrária no Brasil: uma leitura das décadas de 1990 e 2000 (Tesis doctoral). Universidade Federal da Bahia, Programa de Pós-Graduação em Ciências Sociais da Faculdade de Filosofia e Ciências Humanas. Brasil.

Racionero, L. (1986). Sistemas de ciudades y ordenación del territorio. Madrid: Ed. Alianza universidad. 
Segatti, S. M. (2009). A expansão da agroindústria sucroalcooleira e a questão do desenvolvimento da microrregião de Dracena-SP (Trabajo de fin de máster). Universidade Estadual Paulista, Faculdade de Ciências e Tecnologia, Programa de Pós-Graduação em Geografia. Brasil. Recuperado de https:// repositorio.unesp.br/handle/11449/96768

Souza, S.P. de (2007). Assentamentos rurais e novas dinâmicas socioeconômicas: o caso dos municípios de Rosana, Euclides da Cunha Paulista e Teodoro Sampaio-SP (Trabajo de fin de máster). Universidade Estadual Paulista, Faculdade de Ciências e Tecnologia, Programa de Pós-Graduação em Geografia. Brasil. Recuperado de https://repositorio.unesp.br/handle/11449/96756

Secretaria de Administração Penitenciária do Estado de São Paulo (SAP) (2019). Unidades prisionais. Recuperado de http://www.sap.sp.gov.br/uni-prisionais/pen.html

Wanderley, M. de N.B. (2014). O campesinato brasileiro: uma história de resistência. Revista de Economia e Sociologia Rural, 52 (Suppl. 1), 25-44. https://dx.doi.org/10.1590/S0103-20032014000600002

Zárate, M.A. y Rubio, M.T. (2010). Conceptos y prácticas en Geografía Humana. Madrid: Ed. Editorial Universitaria Ramón Areces. 